\title{
OVOS E LARVAS DE HELMINTOS EM CAIXAS DE AREIA DE CRECHES, ESCOLAS MUNICIPAIS E PRAÇAS PÚBLICAS DE CUIABÁ, MT
}

\author{
Valeria Regia Sousa, ${ }^{1}$ Arleana Ferreira Almeida, ${ }^{2}$ Amanda Cavalheiro Cândido ${ }^{3}$ \\ e LuCiano Antunes Barros ${ }^{4}$ \\ 1. Professora de Clínica Médica de Pequenos Animais do Departamento de Clínica Médica Veterinária da Faculdade de \\ Agronomia e Medicina Veterinária da Universidade Federal de Mato Grosso. E-mail: regia@cpd.ufmt.br \\ 2. Mestranda do Curso de Pós-Graduação em Ciências Veterinárias, UFMT \\ 3. Graduanda em Medicina Veterinária, UFMT \\ 4. Professora de Parasitologia Veterinária, UFMT.
}

\section{RESUMO}

As áreas de lazer são de uso frequente na educação infantil. Entretanto, elas podem estar contaminadas por fezes de cães e gatos, predispondo os seus usuários à aquisição de diversas zoonoses. $\mathrm{O}$ presente estudo avaliou a ocorrência dos agentes de larva migrans visceral e cutânea em 20 creches, 36 escolas municipais e 10 praças públicas de Cuiabá, Mato Grosso, no período de junho a novembro de 2006. As amostras de areia e solo foram coletadas em cinco pontos distintos da área de estudo, armazenadas em sacos plásticos e enviadas ao HOVET-UFMT, para imediato processamento através das técnicas de Willis-Mollay, Hoffmann, Pons e Janer e Baermann.
Das instituições pesquisadas, em duas creches, cinco escolas e uma praça pública encontraram-se larvas filariformes e/ou rabtidiformes da área de recreação. O risco de transmissão de larva migrans visceral nas creches estudadas pode ser considerado pequeno, uma vez que não se observou a presença de ovos de Toxocara spp. nas amostras analisadas. Porém, há o risco de infecção das crianças por larva migrans cutânea nesses locais. Dessa forma, há necessidade de desenvolver novos estudos, para determinar as principais fontes de infecção dessa zoonose às crianças matriculadas nas creches e escolas municipais de Cuiabá.

PALAVRAS-CHAVES: Ancylostoma sp., creches, escolas municipais, praças públicas, Toxocara sp.

\section{ABSTRACT}

\section{EGGS AND LARVAE OF HELMINTH IN KINDERGARTENS, PUBLIC SCHOOLS AND PUBLIC SQUARES OF CUIABA, MT}

Playground areas are widely used in elementary and preschool education; however the contamination of these areas by feces of dogs and cats exposes its users to several zoonosis. This study assessed the occurrence of agents of visceral and cutaneous larva migrans at 20 kindergartens, 36 public schools and 10 public squares of Cuiaba, MT, in the period from June to November 2006. The samples of sand and soil were collected at five different points of the study area, stored in plastic bags and sent to the HOVET - UFMT, for immediate processing through the techniques of Willis-Mollay, Hoffmann, Pons and Janer and Baermann. from the institutions surveyed, the institutions surveyed, helminth larvae where found in the recreation areas of two kindergartens, five schools and one public square. The risk of transmission of visceral larva migrans studied in these preschools can be considered small, because there was no presence of Toxocara spp eggs in the samples analyzed. Meanwhile, there is risk of infection of children by larva migrans through skin contact in preschools, elementary schools or the home environment. Thus, further studies should be developed to determine the main sources of infection of zoonosis of children enrolled in preschools and public schools in Cuiabá.

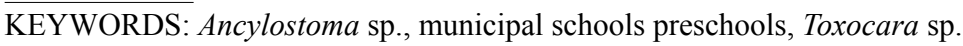




\section{INTRODUÇÃO}

Os animais domésticos, particularmente cães e gatos, possuem um importante papel na sociedade, no que diz respeito ao desenvolvimento emocional de crianças e ao bem-estar de seus proprietários (ALDERETE et al., 2003). Entretanto, esses animais são hospedeiros definitivos para algumas espécies de helmintos, que podem atuar como agentes etiológicos de patologias no organismo humano.

Diversos estudos demonstram que houve uma elevação, no Brasil, do risco de infecção de pessoas por parasitos, principalmente crianças, dado o crescente número de cães domiciliados, peridomiciliados e errantes, de um modo geral, associado ao seu fácil acesso aos locais de lazer (ARAÚJO et al., 2000; NUNES et al., 2000; GUIMARÃES et al., 2005).

Em creches e escolas de ensino infantil, a areia das áreas de lazer pode constituir uma via de transmissão para várias zoonoses parasitárias, representando risco potencial para as crianças que brincam nesses locais. Dentre as espécies de helmintos com potencial zoonótico, encontram-se os agentes etiológicos da larva migrans visceral - Toxocara canis e T. cati; e da larva migrans cutânea - Ancylostoma braziliense e A. caninum (NUNES et al., 2000; GUIMARÃES et al., 2005).

A larva migrans visceral é consequência da ingestão acidental de ovos larvados de Toxocara, eliminados nas fezes de seus hospedeiros naturais, e se caracteriza pela migração das larvas nas vísceras humanas, acarretando casos assintomáticos e processos patológicos hipereosinofílicos crônicos. Segundo CAMPOS JÚNIOR et al. (2003), trata-se de um problema de saúde pública de grande relevância, que não tem sido alvo de estudos e da intervenção necessária para a redução do seu impacto populacional.

A larva migrans cutânea é uma dermatite decorrente do contato direto da pele do ser humano com a larva do terceiro estádio do Ancylostoma spp. Após a penetração na epiderme, as larvas migram no tecido subcutâneo, provocando erupções serpiginosas, distribuídas principalmente nos membros inferiores, pernas, nádegas e mãos (ALMEIDA et al., 2007). No Brasil, essa dermatose tem sido assinalada em vários Estados e frequentemente está relacionada a pacientes que tiveram contato com areia de praias, de depósitos peridomiciliares ou de áreas de recreação (RODRIGUES et al., 2004).

MURADIAN et al. (2005) concluíram que a alta densidade populacional de cães e gatos em áreas urbanas, provavelmente, induz à contaminação do solo, resultando em alta infecção das crianças por Toxocara sp. CAMPOS JUNIOR et al. (2003) verificaram uma frequência de soropositividade para antígenos de $T$. canis sete vezes maior nas crianças que habitavam na periferia de grandes cidades, o que se relaciona com as condições sanitárias do local.

Para MARDER et al. (2004), a existência de parasitos em amostras fecais de cães e gatos que convivem com o homem e sua presença em lugares públicos são fundamentais para interpretar a epidemiologia de inúmeras enfermidades transmissíveis ao homem. Assim, a identificação da contaminação desses locais por ovos ou larvas de helmintos justifica o implemento de medidas preventivas adequadas.

O relato de larva migrans cutânea em crianças de uma escola de educação infantil em Campo Grande, MS, vem alertar sobre o risco de transmissão de zoonoses parasitárias nas áreas de recreação infantil (ARAÚJO et al., 2000).

O objetivo do presente trabalho foi avaliar a ocorrência dos agentes de larva migrans visceral e cutânea nas áreas de recreação das creches, escolas municipais e praças públicas de Cuiabá, MT, por meio da identificação de larvas e/ou ovos de Ancylostoma spp. e de ovos de Toxocara spp.

\section{MATERIAL E MÉTODOS}

No presente estudo foram pesquisadas dezessete creches, dezessete escolas municipais e dez praças públicas de Cuiabá, MT, escolhidas aleatoriamente, no período de junho a novembro de 2006. A mínima amostra requerida neste estudo foi de nove creches e onze escolas, com intervalo de confiança de $95 \%$, calculada pelo programa Epi Info 3.3.2., com uma frequência esperada de 29,7\% (MURADIAN et al., 2005 ) e precisão de $5 \%$, de um total de 26 creches e 79 escolas do município (PREFEITURA MUNICIPAL DE CUIABÁ, 2004).

Durante as visitas, que ocorreram sempre no período da manhã, foram coletadas amostras de fezes e de areia dos parques de recreação. Para a obtenção 
do material a ser analisado, utilizou-se uma pá, sendo realizada a coleta em cinco pontos distintos da área e desprezada a areia superficial, evitando-se a coleta de sujidades. As amostras foram armazenadas em sacos plásticos e enviadas refrigeradas ao Hospital Veterinário da UFMT (HOVET-UFMT) para imediato processamento (SANTOS et al., 2003).

No HOVET-UFMT, as cinco amostras de cada área foram homogeneizadas e tamisadas para obtenção de única amostra (30 gramas), examinadas pelas técnicas de WILLIS-MOLLAY (1921) e HOFFMANN, PONS e JANER (1934), para a obtenção de ovos de helmintos, e a técnica de Baermann para a recuperação de larvas. Para cada amostra, realizaram-se três repetições, nas três técnicas utilizadas. As lâminas obtidas foram examinadas por microscopia óptica em aumento de 10X.

Amostras de fezes que se encontravam sobre o solo foram coletadas e processadas pela técnica de WILLIS-MOLLAY (1921) e HOFFMANN et al. (1934), para a pesquisa de ovos de helmintos, sendo confeccionadas três lâminas em cada técnica e observadas por microscopia óptica.

Informações quanto ao número de crianças, cuidados com as caixas de areia e presença de casos anteriores ou atuais de larva migrans cutânea foram obtidas junto à administração das creches.

\section{RESULTADOS}

Das dezessete creches estudadas, em dezesseis delas foram coletadas amostras de areia e, em uma, amostra de fezes e areia, totalizando dezessete amostras de areia e uma de fezes. Em duas amostras de areia das creches visualizaram-se larvas filariformes e, em apenas uma, rabditiformes. Na amostra de fezes encontrada na areia da área de lazer verificaram-se ovos de Ancylostoma spp.

Nas amostras obtidas nas escolas municipais, em cinco observaram-se larvas de helmintos. Destas, em três havia larvas do tipo rabditiforme e, em uma, do tipo rabditiforme e filariforme. Doze encontravam-se negativas. Em quatro escolas coletaram-se amostras fecais, sendo todas negativas nas técnicas parasitológicas utilizadas.

Uma das dez praças investigadas apresentou larvas filariformes e rabditiformes em sua areia da área de recreação.

A média de alunos das creches visitadas foi de 86,2 e das escolas, 769,1. Segundo dados obtidos junto à administração, em seis $(17,6 \%)$ instituições havia ocorrido casos da doença e em dez $(29,4 \%)$ havia casos anteriores e atuais de larva migrans cutânea nas crianças. De acordo com os dados, em doze instituições informou-se a entrada de cães e gatos $(35,3 \%)$, em sete $(20,6 \%)$ gatos e em uma (2,9\%) cães, sendo estes últimos associados aos pais dos alunos. Em quatorze áreas $(41,2 \%)$, não houve relato da presença dos referidos animais.

Em cinco creches foi informada a troca recente da areia utilizada no parque de recreação infantil. Três dos parques eram novos. Em nenhuma instituição pesquisada havia assistência médica em seu quadro de funcionários, sendo os casos encaminhados aos postos de saúde regionais.

Das sete amostras positivas para larvas de helmintos, a técnica de Baermann detectou cinco e a de Hoffman, duas.

TABELA 1. Ovos e larvas de helmintos em creches, escolas municipais e praças públicas de Cuiabá, MT

\begin{tabular}{ccccc}
\hline \multirow{2}{*}{$\begin{array}{c}\text { Área de estudo } \\
\text { (total/positivos) }\end{array}$} & Oncylostoma sp & Toxocara sp & Filariforme & Larvas \\
\cline { 2 - 5 } Creches (17/3) & 1 & - & 2 & 1 \\
Escolas (17/5) & - & - & 1 & 5 \\
Praças (10/1) & - & - & 1 & 1 \\
\hline Total & 1 & 0 & 4 & 7
\end{tabular}




\section{DISCUSSÃO}

A ocorrência de amostras positivas para larvas do tipo rabditiforme e filariformes é similar à encontrada em trabalho realizado por GUIMARÃES et al. (2005) na cidade de Lavras, MG. Entretanto, o mesmo estudo registrou ovos de Ancylostoma spp. em areia, o que não foi observado nas áreas pesquisadas.

Trabalhos relatam o encontro de ovos de Toxocara spp. em amostras de areia de praças públicas, indicando risco de infecção humana por este agente (CAPUANO \& ROCHA, 2005). Neste trabalho não foram encontrados ovos de Toxocara spp., assim como observado por outros autores (NUNES et al., 2000; GUIMARÃES et al., 2005).

Das amostras de fezes coletadas nas instituições infantis, apenas uma apresentou ovos de Ancylostoma spp. Apesar de não ter sido observada a presença de larvas na referida instituição, isso indica possível contaminação desse ambiente pelo parasito causador da larva migrans cutânea.

$\mathrm{O}$ livre acesso de animais domésticos a áreas de lazer tem sido um dos motivos da maior ocorrência de zoonoses parasitárias. Os gatos foram os principais incriminados por tal contaminação, o que se deve ao maior acesso dessa espécie às caixas de areia, além do hábito de enterrar as fezes, evitando a dessecação e viabilizando a manutenção de tais parasitos no ambiente. $O$ frequente acesso de cães nas praças, inclusive acompanhando os alunos às escolas, propicia a contaminação ambiental por fezes desses animais, podendo gerar uma maior ocorrência de larva migrans visceral e cutânea (ARAÚJO et al., 2000; GUIMARÃES et al., 2005).

Apesar de em apenas duas creches e em cinco escolas terem sido encontradas larvas, havia informações da ocorrência de larva migrans cutânea antes ou durante a realização deste estudo em dezenove instituições estudantis. Segundo avaliação feita pelo 9. ${ }^{\circ}$ Distrito de Metereologia de Cuiabá, MT, o índice pluviométrico dos meses de junho a novembro variou de 4,1 a 142,9 milímetros. A influência dos fatores ambientais, como a umidade e a temperatura média, sobre ovos e larvas dos helmintos, justificaria a baixa prevalência observada, pois no período de realização do estudo é característico na região temperatura elevada com baixa umidade relativa, o que provocaria a dessecação. A ocorrência de casos anteriores de larva migrans cutânea nas crianças provavelmente coincide com a estação chuvosa. Outra hipótese é de a contaminação ser domiciliar, aspecto a ser levado em conta, pois, de acordo com estudo realizado por LEITE et al. (2004), cerca de $45,03 \%$ das amostras fecais de cães de alunos de instituições de ensino apresentaram ovos de helmintos.

Diversos estudos demonstram contaminação das praças públicas por parasitos de caráter zoonótico (COELHO et al., 2001; SANTARÉM et al., 2004; WIWANITKIT \& WAENLOR, 2004; CAPUANO \& ROCHA, 2005; GUIMARÃES et al., 2005; ALMEIDA et al., 2007), principalmente por Ancylostoma spp. e Toxocara spp., associados ao fácil acesso de cães e gatos a esses locais, errantes ou levados pelos proprietários. Esse fato é de extrema importância, sobretudo para crianças, haja vista uma maior predisposição destas para se infectar por tais parasitos, como demonstrado por ALDERETE et al. (2003) e ANARUMA FILHO et al. (2003).

No presente estudo, apenas uma praça pública teve sua areia do parque de recreação contaminada por larvas de helmintos. Entretanto, esse número pode ter sido subestimado, pois o estudo se restringiu às praças onde havia tais parques, não sendo coletadas amostras de solo. Tal justificativa leva em consideração o estudo realizado por ALMEIDA et al. (2007), em que, de 55 praças pesquisadas de Cuiabá, 40 apresentavam amostras fecais caninas, de um total de 121 amostras coletadas, sendo 45 delas contaminadas por ovos de helmintos. Esse dado demonstra que há uma maior contaminação das praças públicas de Cuiabá que a obtida no presente estudo.

Em análise das técnicas utilizadas na verificação das amostras obtidas, apesar de não ter ocorrido diferença significativa entre as três, pode-se obter um resultado mais fidedigno da contaminação desses locais, com a associação delas, elevando para sete o número de instituições contaminadas.

Segundo GUIMARÃES et al. (2005), a frequência de larvas de helmintos observada em ambientes de recreação sugere que, provavelmente, não são adotadas medidas restritivas rígidas para controle da circulação de animais nesses locais. A simples troca da areia de áreas de recreação de praças públicas não é suficiente para controlar a contaminação por ovos de helmintos 
de cães e gatos, o que foi conseguido cobrindo as caixas de areia com lonas durante a noite (ARAÚJO et al., 2000; SANTARÉM et al., 2004). Essa precaução não foi observada em nenhuma das creches e escolas visitadas, e a difícil execução de tais medidas nas praças públicas pode incrementar ainda mais o risco de contaminação.

Segundo RODRIGUES et al. (2004), a pesquisa de ovos/larvas de helmintos cada vez que a areia da área de recreação for trocada é fortemente recomendada, dada a possibilidade de contaminação dessa área com fezes de cães e gatos nos depósitos das lojas de material de construção. Esse é um dado que não foi relatado em nenhum local visitado. Além disso, as trocas dessas areias eram realizadas em apenas cinco $(14,7 \%)$ instituições.

Não foi possível verificar o fator de risco deste estudo, dada a indisponibilização de diagnóstico definitivo dos alunos relatados com larva migrans cutânea nas creches e escolas. Essa informação de casos positivos anteriores e/ou atuais da infecção em dezesseis $(47,1 \%)$ instituições pesquisadas foi obtida por parte da diretoria das instituições públicas, que não soube precisar o local de aquisição da enfermidade, se no ambiente domiciliar ou escolar.

A realização de programas integrados de educação sanitária, visando ampliar os conhecimentos dos professores de educação infantil sobre os aspectos relacionados à aquisição de zoonoses (TOME et al., 2005), poderia fornecer dados mais fidedignos da doença, bem como medidas adequadas de controle e tratamento de diversas enfermidades.

Outro fator relevante no controle das referidas zoonoses refere-se à educação dos proprietários de cães e gatos, evitando o acúmulo de seus dejetos em ambientes públicos e o acesso a centros educacionais, além da administração periódica de anti-helmínticos sob a orientação de médicos veterinários.

\section{CONCLUSÃO}

O risco de transmissão de larva migrans visceral nas creches estudadas pode ser considerado pequeno, uma vez que não se observou a presença de ovos de Toxocara spp. nas amostras de areia analisadas. Entretanto, há risco de infecção das crianças por larva migrans cutânea, seja nas creches, escolas ou em pra- ças públicas. Dessa forma, novos estudos devem ser desenvolvidos para determinar as principais fontes de infecção dessa zoonose para as crianças matriculadas nas creches e escolas municipais de Cuiabá.

\section{REFERÊNCIAS}

ALDERETE, J. M. S.; JACOB, C. M. A.; PASTORINO, A. C.; ELEFANT, G. R.; CASTRO, A. P. M.; FOMIN, A. B. F.; CHIEFFI, P. P. Prevalence of Toxocara infection in schoolchildren from the Butantã Region, São Paulo, Brazil. Memórias do Instituto Oswaldo Cruz, v. 98, n. 5, p. 593-597, 2003.

ALMEIDA, A. B. P. F.; SOUSA, V. R. F.; DALCIN, L.; JUSTINO, C. H. S. Contaminação por fezes caninas das praças públicas de Cuiabá, Mato Grosso. Brazilian Journal Veterinary Research and Animal Science, v. 44, n. 2, p.132-136, 2007.

ANARUMA FILHO, F.; CHIEFFI, P. P.; CORREA, C. R. S.; CAMARGO, E. D.; SILVEIRA, E. P. R.; ARANHA, J. J. B. Human Toxocariasis: incidence among residents in the outskirts of Campinas, State of São Paulo, Brazil. Revista do Instituto de Medicina Tropical de São Paulo, v. 45, n. 5, p. 293-294, 2003.

ARAÚJO, F. R.;ARAÚJO, C.P.; WERNECK, M. R.; GÓRSKI,A. Larva migrans cutânea em crianças de uma escola em área do Centro-Oeste do Brasil. Revista de Saúde Pública, v. 34, n. 1, p. 84-85, 2000.

CAMPOS JÚNIOR, D.; ELEFANT, G. R.; MELO E SILVA, E. O.; GANDOLFI, L.; JACOB, C. M. A.; TOFETI, A.; PRATESI, R. Freqüência de soropositividade para antígenos de Toxocara canis em crianças de classes sociais diferentes. Revista da Sociedade Brasileira de Medicina Tropical, v. 36, n. 4, p. 509-513, 2003.

CAPUANO, D. M.; ROCHA, G. M. Environmental contamination by Toxocara sp. eggs in Ribeirão Preto, São Paulo State, Brazil. Revista do Instituto de Medicina Tropical de São Paulo, v. 47, n. 4, p. 223-226, 2005.

COELHO, L. M. D. P. S.; DINI, C. Y.; MILMAN, M. H. S. A.; OLIVEIRA, S. M. Toxocara spp. eggs in public squares of Sorocaba, São Paulo State, Brazil. Revista do Instituto de Medicina Tropical de São Paulo, v, 43, n. 4, p. 189-191, 2001.

HOFFMAN, W. A.; PONS, J. A.; JANER, J. L. The sedimentation concentration method in Schistosomiasis mansoni. Journal of Public Health, v. 9, p. 281-289, 1934.

GUimarÃES, A. M.; ALVES, E. G. L.; REZENDE, G. F.; RODRIGUES, M. C. Ovos de Toxocara sp. e larvas de Ancylostoma sp. em praça pública de Lavras, MG. Revista de Saúde Pública, v. 39, n. 2, p. 293-295, 2005. 
LEITE, L. C.; MARINONI L. P.; CÍRIO, S. M.; DINIZ, J. M. F.; SILVA, M. A. N.; LUZ, E.; MOLINARI, H. P.; VARGAS, C. S. G.; LEITE, S. C.; ZADOROSNEI, A. C. B.; VERONESI, E. M. Endoparasitas em cães (Canis familiares) na cidade de Curitiba - Paraná - Brasil. Archives of Veterinary Science, v. 9, n. 2, p. 95-99, 2004.

MARDER, G.; ULON, S. N.; BOTTINELLI, O. R.; MEZA FLEITAS, Z.; LOTERO, D. A.; RUIZ, R.; PEIRETTI, H. A.; ARZÚ, R. A. Infestación parasitaria en suelos y materia fecal de perros y gatos de la ciudad de Corrientes. Revista Veterinaria, v. 15, n. 2, p. 70-72, 2004.

MURADIAN, V.; GENNARI, S. M.; GLICKMAN, L. T.; PINHEIRO, S. R. Epidemiological aspects of Visceral Larva Migrans in children living at São Remo Community, São Paulo (SP), Brasil. Veterinay Parasitology, v. 134, p. 93-97, 2005.

NUNES, C. M.; PENA, F. C.; NEGRELLI, G. B.; ANJO, C. G. S.; NAKANO, M. M.; STOBBE, N. S. Ocorrência de larva migrans na areia de áreas de lazer das escolas municipais de ensino infantil, Araçatuba, SP, Brasil. Revista de Saúde Pública, v. 34, n. 6, p. 656-658, 2000.

PREFEITURA MUNICIPAL DE CUIABÁ. Perfil socioeconômico de Cuiabá, v. II. Cuiabá: Instituto de Pesquisa e Desenvolvimento Urbano, 2004. 407 p.

RODRIGUES, M. M.; ARAÚJO, A.; MACHADO, D.; FONSECA, F. A. R.; JÚNIOR, M. A. R. A importância das condições de higiene em áreas de recreação infantil. Disponível em: $<$ http:// www.ufmg.br/congrext/Saude/Saude4.pdf $>$. Acesso em: 15 jan. 2008.

SANTARÉM, V. A.; GIUFFRIDA, R.; ZANIN, G. A. Larva migrans cutânea: ocorrência de casos humanos e identificação de larvas de Ancylostoma spp. em parque público do município de Taciba, São Paulo. Revista da Sociedade Brasileira de Medicina Tropical, v. 37, n. 2, p. 179-181, 2004.

SANTOS, R. S.; BONATO, L. C.; MARQUES, M. P. A. Ocorrência de agentes causais de larva migrans em areias destinadas à recreação em escolas de ensino infantil em Espírito Santo do Pinhal, SP, Brasil. Revista Ecossistema, v. 28, n. 1, 2, p. 57-60, 2003.

TOME, R. O.; SERRANO, A. C. M.; NUNES, C. M.; PERRI, S. H. V.; BRESCIANI, K. D. S. Inquérito epidemiológico sobre conceitos de zoonoses parasitárias para professores de escolas municipais do ensino infantil de Araçatuba-SP. Revista Ciência em Extensão, v. 2, n. 1, p. 38-46, 2005.

WILLIS, H. H. A simple levitation method for the detection of wookworm ova. Medicine Journal of Australia, v. 8, p. 375376, 1921.

WIWANITKIT, V.; WAENLOR, W. The frequency rate of Toxocara species contamination in soil samples from public yards in a urban area "Payathai", Bankok, Thailand. Revista do Instituto de Medicina Tropical de São Paulo, v. 46, n. 2, p. 113-114, 2004.

Protocolado em: 17 jan. 2008. Aceito em: 5 out. 2009. 